\title{
Types of Renal Calculi and Management Regimen for Chinese Minimally Invasive Percutaneous Nephrolithotomy
}

\author{
Si-Ping Gu • Guo-Hua Zeng • Zhi-Yuan You • Yi-Jin Lu • \\ Yun-Teng Huang • Qing-Mao Wang • Zhao-Hui He
}

Received: 14 September 2013 / Accepted: 10 February 2014 / Published online: 20 March 2014

(C) Association of Surgeons of India 2014

\begin{abstract}
Strict selection of patients for minimally invasive percutaneous nephrolithotomy could effectively improve the success rate of surgery. This study aimed to understand the required skills and the efficacy of miniPCNL in the treatment of five types of upper ureteral calculi. Data collected after X-ray analysis and B mode ultrasound from 633 patients with upper ureteral and renal pelvis calculi who underwent B ultrasound-guided lithotomy was reviewed, including the following: type I, upper ureteral or renal pelvis calculi with moderate hydronephrosis (154 cases); type II, upper ureteral or renal pelvis calculi with severe hydronephrosis (157 cases); type III, upper ureteral or renal pelvis calculi without hydronephrosis (61 cases); type IV, renal pelvis calculi, one or two renal calyx calculi (206 cases); and type $\mathrm{V}$, renal staghorn calculi (55 cases). Operations on 611 cases were successful. The treatment method for five patients was converted to open surgery. Twelve cases were treated by indwelling double-J tube retrocatheterization and extracorporeal shock wave lithotripsy. Five patients gave up the treatment. The rate of calculus clearance was $82.3 \%$, and the rate of residual calculus was $17.6 \%$. Selective renal artery embolization was
\end{abstract}

Si-Ping Gu and Guo-Hua Zeng are co-first authors.

S.-P. Gu·Z.-Y. You • Q.-M. Wang

Department of Urology, Xinhua Hospital Affiliated to Shanghai Jiao

Tong University School of Medicine, No. 1665 Kongjiang Road

Yangpu District, 200092 Shanghai, China

G.-H. Zeng $•$ Y.-J. Lu • Y.-T. Huang $(\bowtie) \cdot$ Z.-H. He

Department of Minimally Invasive Surgery, Shishi Hospital,

362700 Shishi, Fujian Province, China

e-mail: yuntenghuangen@163.com performed in nine cases. Hydropneumothorax occurred in nine cases. No intestinal fistula occurred, and no patient had to undergo nephrectomy. The difficulty and the curative effect of the operation were different because the types of calculi varied. Selection of the procedure based on the different types of calculi could effectively improve the success rate of the procedure, reduce complications, and shorten the learning curve.

Keywords Minimally invasive nephrolithotomy - Types of calculus $\cdot$ Upper ureteral and renal calculi $\cdot$ Renal staghorn calculi $\cdot$ Hydronephrosis

\section{Introduction}

Minimally invasive percutaneous nephrolithotomy (miniPCNL) is one of the common methods used to treat upper ureteral calculus. However, the difficulty of the operation may vary, and the learning curve may be longer because calculi are of different types [1-3]. Serious complications such as intraoperative bleeding and nephrectomy have also been reported [4-9]. Data from 633 patients with upper ureter calculus treated by miniPCNL from 2006 to 2009 were reviewed in this study. The upper ureteral and renal pelvis calculi were divided in five types according to the site, size, shape, degree of hydronephrosis in the renal collecting system, and anatomical characteristics. Surgery difficulty was observed to increase depending on the type of calculi. The treatment effect was satisfactory, and no serious complications were observed. 


\section{Data and Methods}

\section{General Data}

A total of 633 patients comprising 336 males and 297 females aged 12 to 85 years (average age $=41$ years) were studied. Ipsilateral discomfort or pain in the renal region, renal colic history, fever, and hematuria history were observed in 601 , 189 , and 103 patients, respectively. A total of 16 patients were pyonephrotic, whereas 32 patients were asymptomatic. Patients with anatomical kidney abnormalities, such as solitary kidney, renal duplication, and colon in the back of the kidney, were excluded in this study. Urinary B ultrasound, KUB + IVP, and bilateral renal CT plus three-dimensional reconstruction were conducted before the operation. The patients were grouped according to the site of calculus: 202 cases with calculi in the upper ureteral segment or the ureteropelvic junction (UPJ), 123 cases with pelvic calculi, 6 cases with upper calyx calculi, 9 cases with middle calyx calculi, 5 cases with lower calyx calculi, 30 cases with more than two calyceal calculi, 181 cases of renal pelvis calculi combined with calyceal calculi, and 55 cases of renal staghorn calculi. The sizes of the upper ureteral calculi ranged from 0.8 to $3.0 \mathrm{~cm}$ (average $=1.9 \mathrm{~cm}$ ), whereas the sizes of the renal calculi ranged from 1.5 to $6.5 \mathrm{~cm}$ (average $=2.2 \mathrm{~cm}$ ); 102 patients underwent preoperative extracorporeal shock wave lithotripsy (ESWL). Sixty-nine patients with upper urinary calculi underwent open surgery; 103 cases had calculi in both kidneys. Severe, moderate, and mild calyceal hydronephrosis were observed in 158, 161, and 134 patients, respectively. In order to decrease bleeding during operation and postoperation, hypertension was controlled to less than 140/ $90 \mathrm{mmHg}$. To reduce infection tendency, the concentration of blood sugar in patients with diabetes was controlled to less than $11.2 \mathrm{mmol} / \mathrm{L}$. This study was conducted in accordance with the Declaration of Helsinki. This study was conducted with approval from the Ethics Committee of Shanghai Jiao Tong University School of Medicine. Written informed consent was obtained from all participants.

\section{Types of Calculus}

In this study, the calculi were divided in five types according to the site, size, shape, and obstruction. Type I comprised the upper ureteral or renal pelvis calculi with moderate hydronephrosis (154 cases). The thickness of the renal parenchyma was $>1.5 \mathrm{~cm}$. Type II comprised the upper ureteral or renal pelvis calculi with severe hydronephrosis (157 cases). The thickness of the renal parenchyma was $<1.0 \mathrm{~cm}$. Type III comprised the upper ureteral or renal pelvis calculi without hydronephrosis (61 cases). The thickness of the renal parenchyma was normal. Type IV comprised the renal pelvis calculi and one or two renal calyx calculi (206 cases). Type V comprised the renal staghorn calculi in multiple renal calices except in the renal pelvis (55 cases).

\section{Operation Procedures}

Patients were anesthetized and put in lithotomy position, and an F5 ureteral catheter was passed retrograde using a ureteroscope. Afterward, the patients were moved to a prone position. Under the guidance of a C-arm X-ray or highfrequency $\mathrm{B}$ ultrasound, the target renal calyx was punctured with an 18-gauge renal paracentetic needle and a kidney puncture nephrostomy kit (COOK, USA). After the target was successfully punctured, a Zebra guidewire was placed into the calyx, ureter, or renal pelvis through the needle sheath. The channels from F8 to F18-20 were sequentially expanded using a fascia dilator to establish the percutaneous removal channel of the renal calculi. The operation was performed by a Richard Wolf lithotripsy (Germany), Swiss ultrasonic lithotripsy (EMS-III), or holmium laser lithotripsy. Another channel was established via the target puncture as described above to clean the residual calculi. An F5 double-J tube was placed indwelling in the ureter and a nephrostomy tube in the nephrostomy port. Plain abdominal radiograph or CT was repeated after the operation. For cases with more residual calculi, the second or third mini-PCNL was performed 5 to 7 days after the previous operation [10-13].

\section{Results}

The tunnel was successfully established in 611 patients, but failed in 22 cases mainly because of unsuccessful puncture (6 cases), prolapse of the Zebra guidewire caused by the loss of a channel (4 cases), and displacement of the established channel from the expected renal calices ( 2 cases). A double-J tube was transurethrally indwelled, and ESWL was performed in 12 patients. Five patients with failed puncture refused to undergo open surgery and were transferred to another hospital for treatment. The other three cases underwent open surgery because of larger calculi and severe obstruction or contralateral renal agenesis. Two cases underwent open surgery because the target was unsuccessfully punctured (Table 1). A tunnel was successfully established in 474, 102, and 35 patients at stages I, II, and III of the operation (PCNL), respectively. Single-, dual-, and triple-channel procedures were conducted on 481, 112, and 18 patients, respectively. Residual calculi ( $\geq 0.5 \mathrm{~cm}$ in diameter) were observed in 22 patients. A total of 12 cases of postoperative ESWL, 9 cases of postoperative hydropneumothorax, and 9 cases of arteriovenous fistula followed by treatment of interventional embolization were observed. No leaky gut, nephrectomy, or deaths occurred among any of the patients. The results of mini-PCNL 
Table 1 Success rate of channel establishment and failure treatment

\begin{tabular}{lllll}
\hline Types & Success & Failure & & \\
\cline { 3 - 5 } & & $\begin{array}{l}\text { Double-J } \\
\text { tube + ESWL }\end{array}$ & $\begin{array}{l}\text { Conversion to } \\
\text { open surgery }\end{array}$ & $\begin{array}{l}\text { Treatment } \\
\text { abandonment }\end{array}$ \\
\hline I $(n=154)$ & 153 & 1 & 0 & 0 \\
II $(n=157)$ & 151 & 2 & 3 & 1 \\
III $(n=61)$ & 52 & 6 & 1 & 2 \\
IV $(n=206)$ & 200 & 3 & 1 & 2 \\
V $(n=55)$ & 55 & 0 & 0 & 0 \\
Total 633 & 611 & 12 & 5 & 5 \\
\hline
\end{tabular}

performed to treat the five types of the upper ureteral and renal pelvis calculi are shown in Table 2.

\section{Discussion}

Mini-PCNL surgery has a longer learning curve and may result in serious complications such as massive intraoperative and postoperative hemorrhages. This surgery may even lead to nephrectomy. Thus, the aforementioned procedure is difficult and requires great skill [1-9]. In this study, the operations started from simple surgical cases (depending on the calculus type) to help these surgeons gain experience. More difficult cases were gradually handled, thereby improving the success rate of the surgery, reducing complications and the rate of residual calculus, and shortening the learning curve.

The target was easily punctured because the renal collecting system was expanded in 153 cases with type I calculi. Thus, this group was simpler because of a small calculus burden and shorter operative time. The first calculus clearance rate in type I was $92.2 \%(141 / 153)$ with an average operation time of $45 \mathrm{~min}$. Thus, mini-PCNL should be performed for type I calculi. The ideal channel could be established mostly at the 11th intercostal space on the left side of the patient at the subcostal space, or at the 11th intercostal space on the right side of the patient. Puncture and channels were easily established in 151 cases with type II calculi.
However, the anatomical structure of the renal collecting system significantly changed because of various calyceal expansions caused by hydronephrosis. UPJ was not easily located in the first 20 cases at an early stage because the miniPCNL surgeons lacked skills. Thus, methylene blue was retrogradely injected into the indwelling ureteral catheter to find UPJ. Pyonephrosis was found in 11 cases of this group after puncture, and ostomy was performed to construct drainage in the first phase of the operation. Re-lithotripsy was subsequently performed in the second phase. No aggravated infection and diffusion were noted, and this finding is consistent with that of previous studies $[14,15]$. Postoperative renal arteriovenous fistula was formed in one case and cured by selective renal artery embolization. No obvious causal relationship was noted between the formation of arteriovenous fistula and the thickness of the renal parenchyma which commonly related to the degree of hydronephrosis. The expansion of a puncture to the sites of the calyx neck should be avoided, and this finding is consistent with that in a previous study [8]. The first calculus clearance rate among the type II cases was $92.1 \%(139 / 151)$, and the average operation time was $52 \mathrm{~min}$. The surgical operation for type II cases was more difficult than that for type I cases. Establishment of the channels was quite difficult for the 52 cases in type III because renal hydronephrosis was absent and space constraints were observed. The localization of negative calculi by X-ray was also difficult, causing a greater difficulty on the operation and resulting in a higher failure rate of the puncture. The kidney outline, the position of the calculi, and the predetermined path from the insertion point to the pelvis or calculi were detected only by B ultrasound. After the target was successfully punctured, antegrade contrast study of the renal collecting system was performed to determine whether or not the puncture point was the targeted renal calyx. The first and the second calculus clearance rates among the type III cases were $69.2 \%(36 / 52)$ and $30.8 \%(16 / 52)$, respectively. Mini-PCNL in type III was categorized to have middle difficulty. Greater difficulty was experienced in the 200 type IV cases because of the presence of calculi in two renal calices. Calculi are difficult to clean through a single channel under a small angle of renal calices; thus, the expected purpose of the operation could be achieved

Table 2 Treatment results for the five different types of urinary tract stones by mini-PCNL

\begin{tabular}{|c|c|c|c|c|c|c|c|c|}
\hline Types & $\begin{array}{l}\text { Operation } \\
\text { time (min) }\end{array}$ & $\begin{array}{l}\text { Stone clearance } \\
\text { rate for the first } \\
\text { time }(\%)\end{array}$ & $\begin{array}{l}\text { Second } \\
\text { phase of } \\
\text { PCNL }\end{array}$ & $\begin{array}{l}\text { Final rate } \\
\text { of residual } \\
\text { stones }(\%)\end{array}$ & $\begin{array}{l}\text { Decrease in amount of } \\
\text { hemoglobin } 48 \mathrm{~h} \text { after } \\
\text { the operation }(\mathrm{g} / \mathrm{L})\end{array}$ & $\begin{array}{l}\text { Postoperative } \\
\text { fever }\end{array}$ & $\begin{array}{l}\text { Selective renal } \\
\text { artery } \\
\text { embolization }\end{array}$ & Hydropneumothorax \\
\hline $\mathrm{I}(n=153)$ & 20 95 (45) & $92.2(141 / 153)$ & 12 & $5.2(8 / 153)$ & $1 \sim 2(1.2)$ & 5 & 2 & 2 \\
\hline II $(n=151)$ & $30 \sim 102(52)$ & $90.1(136 / 151)$ & 15 & $7.9(12 / 151)$ & $1 \sim 2.1(1.1)$ & 5 & 1 & 1 \\
\hline III $(n=52)$ & $60 \sim 132(93)$ & $69.2(36 / 52)$ & 16 & $13.5(7 / 52)$ & $1.2 \sim 3.1(2.0)$ & 3 & 1 & 2 \\
\hline IV $(n=200)$ & $78 \sim 212$ (103) & $68(136 / 200)$ & 64 & $16.0(32 / 200)$ & $1.5 \sim 3.5(2.6)$ & 2 & 2 & 1 \\
\hline $\mathrm{V}(n=55)$ & $120 \sim 286(215)$ & $0(0 / 55)$ & 55 & $89.1(49 / 55)$ & $2 \sim 5(3.0)$ & 10 & 3 & 3 \\
\hline
\end{tabular}


by constructing two channels and performing the second stage of the operation. The back set of the middle renal calices or the renal calices with hydronephrosis could be considered as the first puncture target to establish the main channel used to extract the calculi. The establishment of the secondary channel often requires the guidance of a $\mathrm{C}$-arm X-ray machine or a $\mathrm{B}$ ultrasound. Hydropneumothorax likely formed as the channel was established at the 10th or the 11th intercostal space. B ultrasound positioning can be used to avoid damage to the lungs, liver, spleen, colon, or blood vessels as well as to prevent the formation of postoperative hydropneumothorax $[9,11]$. The second and the third calculus clearance rates in the type IV cases were $32 \%(64 / 200)$ and $7.5 \%(15 / 200)$, respectively. The rate of residual calculi was $16 \%(32 / 200)$. Therefore, the operation for type IV calculi should be performed after types I, II, and III were skillfully operated and a certain number of operations were recorded. As the renal pelvis and calices were filled with the calculi, a better therapeutic effect could be achieved by forming multiple channels and performing two or three stages of surgery. The operation was likely more difficult if the renal calices were branched and their diameter angles were relatively small. The first channel was typically formed in the middle or the upper calices. After the main calculi were treated, the contrast agent was injected to develop contrast images of the renal pelvis and calices. Xray or B ultrasound was used to establish the second and the third channels for the calculi of the renal calices oriented at an acute angle with the main channel. A secondary channel can be established completely or partially. EMS ultrasonic lithotripsy systems currently used are more efficient. In this system, the residual rate of the calculi can be reduced because of the adsorption function during the lithotripsy process. The average operation time in this type was $215 \mathrm{~min}$. The rate of residual calculi $(\geq 0.5 \mathrm{~cm}$ in diameter) was $89.1 \%(49 / 55)$. The treatment for this group of cases had the highest difficulty of operation, the longest operative time, and the highest rate of residual calculus. Surgical experience should be further summarized because the number of cases in this group was fewer. Failure of the operation in 22 of the 633 cases was caused by different factors. Five cases had unsuccessful puncture operation and refused to proceed with the proposed conversion to open surgery. Three cases (two type II case and one type III case) underwent open surgery because of larger calculi, severe obstruction, or contralateral renal agenesis. Two cases (one case from types II and III) agreed to convert to open surgery after puncture failed. The operation failed in the other 12 cases (one type I, two type II, six type III, and three type IV cases), resulting in unsuccessful puncture (6 cases), prolapse of the Zebra guidewire caused by the loss of a channel ( 4 cases), and displacement of the established channel from the expected renal calices ( 2 cases). The calculi were transurethrally pushed into the kidney by using a ureteroscope with the patients in Trendelenburg position. A double-J tube was indwelled, and
ESWL surgery was performed in the 12 cases. We realized that the aforementioned conditions could be avoided by gaining technical experience on the mini-PCNL combined with the intraoperative use of X-ray and B ultrasound.

The following results were observed from the mini-PCNL surgery in the five types of calculi. Type I and II cases had calculi in the upper ureteral, UPJ, or pelvic calculi combined with middle/severe hydronephrosis. The calculi could be satisfactorily removed with X-ray or B ultrasound by puncturing the renal middle calyx at the 11th intercostal or the subcostal space [10-12]. Although this puncture operation is simple, the upper calyx calculi can be easily removed because the channel is established through the lower calyx. The upper ureteral or UPJ calculi are also difficult to clean. Large mirror swing amplitude may tear a very thin renal parenchyma. MiniPCNL is more difficult to perform at the early or middle stage in type III cases as there was no hydronephrosis and thereby little space. After a target was successfully punctured guided by X-ray or B ultrasound, the contrast agent could be injected using a needle to identify whether or not the agent is in the target renal calyx and the needle is in the renal calyx neck, thereby reducing the incidence of postoperative arteriovenous fistula $[16,17]$. The presence of multiple and complicated calculi in types IV and V cases results in a more difficult surgical treatment and relatively high surgical risk as well as incidence of complications. The following guidelines should be considered. (1) Adequate preoperative and postoperative anti-infection treatment should be administered. Renal calyces filled with this type of calculi for a long period may result in poor urine drainage and bacterial growth in the renal calyces as well as in the calculi. Sensitive antibiotics should be selected carefully before and after surgery. In these types of patients, preoperative sensitive antibiotics should be used for 5-7 days, and it should last to third day after the patient becomes afebrile $[14,15]$. (2) The location and the number of puncture channels should be carefully selected. The designed main channel can be used to process the renal calyceal calculi in addition to the pelvic calculi. The angle between the main channel and the renal calyx of the residual calculi was small, and the residual calculi cannot be clearly extracted. Thus, the sub-channel can be established under the guidance of X-ray and B ultrasound. Each renal calyx was filled with the staghorn calculi in type $\mathrm{V}$ cases, thereby requiring a complete understanding of the distribution of the stones in the renal pelvis, calyces, and front as well as rear groups of calyces. Two or more channels in this type should be established conventionally to complete the operation, thereby resulting in the highest residual rate. The use of an ultrasonic lithotripter (EMS) can significantly improve the efficiency of stone extraction and reduce the residual stones as well as the number of the established channels $[10$, 12, 13]. (3) Perfusion pressure should be maintained at the lowest possible rate during operation, and the operation time should be strictly controlled. The perfusion pressure should be 
maintained at $<30 \mathrm{mmHg}$ to prevent intraoperative absorption of bacteria and toxins as well as to avoid the occurrence of postoperative fever and urinary sepsis $[18,19]$. In addition, the outflow speed of the lavage fluid should be closely monitored during the operation because the nephrostomy channel selected in the micro-PCNL was $<24 \mathrm{Fr}$, and the gap between the peel-away sheath and 8/9.8 Fr of the ureteroscope was relatively small. In case of a sluggish outflow of the lavage fluid caused by stone clogging, the ureteroscope should be pulled out to clear the residual stones [19, 20]. An operation time of $2 \mathrm{~h}$ should be strictly observed because "time is bleeding," that is, longer operation may result in bleeding at a large extent and induce absorption of intraoperative liquid and toxins, thereby causing postoperative complications [16].

Different types of upper urinary tract calculi cause various mini-PCNL operational difficulties, operative complications, and efficacies of stone extraction methods. Selection of the procedure based on the type of calculi can effectively improve the success rate of the procedure, reduce complications, and shorten the learning curve.

Acknowledgement This study was supported by the 2013 Project from the Science and Technology Bureau, Quanzhou City, Fujian Province, China; 2010 Talents Project from the Organization Department, Quanzhou City, Fujian Province, China; and 2013 Project from the Science and Technology Bureau, Shishi City, Fujian Province, China No.2013SK015).

Ethics Committee Approval This study was conducted with approval from the Ethics Committee of Shanghai Jiao Tong University School of Medicine. Written informed consent was obtained from all participants.

\section{References}

1. Tanriverdi O, Boylu U, Kendirci M, Kadihasanoglu M, Horasanli K, Miroglu C (2007) The learning curve in the training of percutaneous nephrolithotomy. Eur Urol 52:206-211

2. de la Rosette JJ, Laguna MP, Rassweiler JJ, Conort P (2008) Training in percutaneous nephrolithotomy - a critical review. Eur Urol 54: 994-1001

3. Allen D, O'Brien T, Tiptaft R, Glass J (2005) Defining the learning curve for percutaneous nephrolithotomy. J Endourol 19:279-282

4. Mitropoulos D, Artibani W, Graefen M, Remzi M, Rouprêt M, Truss M (2012) Reporting and grading of complications after urologic surgical procedures: an ad hoc EAU guidelines panel assessment and recommendations. Eur Urol 61:341-349

5. Seitz C, Desai M, Häcker A, Hakenberg OW, Liatsikos E, Nagele U, Tolley D (2012) Incidence, prevention, and management of complications following percutaneous nephrolitholapaxy. Eur Urol $61: 146-158$

6. Skolarikos A, de la Rosette J (2008) Prevention and treatment of complications following percutaneous nephrolithotomy. Curr Opin Urol 18:229-234

7. Srivastava A, Singh KJ, Suri A et al (2005) Vascular complications after percutaneous nephrolithotomy: are there any predictive factors? Urology 66:38-40

8. Mathieu J, Schulman CC, Struyven J (1978) Percutaneous embolic occlusion of an acquired bleeding intrarenal aneurysm. Eur Urol 4: 212-213

9. Boon JM, Shinners B, Meiring JH (2001) Variations of the position of the colon as applied to percutaneous nephrostomy. Surg Radiol Anat 23:421-425

10. Osman M, Wendt-Nordahl G, Heger K, Michel MS, Alken P, Knoll T (2005) Percutaneous nephrolithotomy with ultrasonographyguided renal access: experience from over 300 cases. BJU Int 96: $875-878$

11. Basiri A, Ziaee AM, Kianian HR, Mehrabi S, Karami H, Moghaddam SM (2008) Ultrasonographic versus fluoroscopic access for percutaneous nephrolithotomy: a randomized clinical trial. $\mathrm{J}$ Endourol 22:281-284

12. Falahatkar S, Neiroomand H, Enshaei A, Kazemzadeh M, Allahkhah A, Jalili MF (2010) Totally ultrasound versus fluoroscopically guided complete supine percutaneous nephrolithotripsy: a first report. J Endourol 24:1421-1426

13. Feng MI, Tamaddon K, Mikhail A, Kaptein JS, Bellman GC (2001) Prospective randomized study of various techniques of percutaneous nephrolithotomy. Urology 58:345-350

14. Zanetti G, Paparella S, Trinchieri A, Prezioso D, Rocco F, Naber KG (2008) Infections and urolithiasis: current clinical evidence in prophylaxis and antibiotic therapy. Arch Ital Urol Androl 80: 5-12

15. Gonen M, Turan H, Ozturk B, Ozkardes H (2008) Factors affecting fever following percutaneous nephrolithotomy: a prospective clinical study. J Endourol 22:2135-2138

16. Tomaszewski JJ, Smaldone MC, Schuster T, Jackman SV, Averch TD (2010) Factors affecting blood loss during percutaneous nephrolithotomy using balloon dilation in a large contemporary series. J Endourol 24:207-211

17. Turna B, Nazli O, Demiryoguran S, Mammadov R, Cal C (2007) Percutaneous nephrolithotomy: variables that influence hemorrhage. Urology 69:603-607

18. Zhong W, Zeng G, Wu K, Li X, Chen W, Yang H (2008) Does a smaller tract in percutaneous nephrolithotomy contribute to high renal pelvic pressure and postoperative fever? J Endourol 22:21472151

19. Troxel SA, Low RK (2002) Renal intrapelvic pressure during percutaneous nephrolithotomy and its correlation with the development of postoperative fever. J Urol 168:1348-1351

20. Nagele U, Horstmann M, Sievert KD et al (2007) A newly designed amplatz sheath decreases intrapelvic irrigation pressure during mini-percutaneous nephrolitholapaxy: an in-vitro pressure-measurement and microscopic study. J Endourol 21: $1113-1116$ 